Jahangirnagar University J. Biol. Sci. 9(1 \& 2): 91-108, 2020 (June \& December)

\title{
Floristic composition and plant species diversity in and around the brickfield areas of Savar-Dhamrai region of Dhaka district
}

\author{
Saiful Islam*, Shayla Sharmin Shetu, Md. Abdur Rahim and Saleh Ahammad Khan \\ Department of Botany, Jahangirnagar University, Savar, Dhaka-1342, Bangladesh
}

\begin{abstract}
This study has confirmed the occurrence of a total of 181 species of vascular plants under 133 genera and 51 families and estimates the plant species diversity in and around the brickfield areas of Savar-Dhamrai region of Dhaka district. Of these, six species were pteridophytes, 175 were Angiosperms, and 144 species were economically important. Five families, composed of six species, belonged to Pteridophyta, 40 families, consist of 128 species, to Magnoliopsida (dicotyledons) and six families, comprised of 47 species, to Liliopsida (monocotyledons). Total of 161 species were herbs, 14 were shrubs, and only six were trees. Asteraceae with 14 species and Poaceae with 24 species were the largest family in Magnoliopsida and Liliopsida, respectively. Amaranthus L., Persicaria (L.) Mill., Solanum L. and Lindernia All. with four species each were appeared as the best representative genera in Mangoliopsida, while Cyperus L. with five species in Liliopsida. The species composition and diversity of abandoned brickfields were found to be higher than those of functional brickfields. A total of 42 (23.20\%) species were common in both abandoned and functional brickfields of this region. Species composition in the abandoned brickfields of Savar and Dhamrai was mostly similar (64.15\%). This study suggests to control and monitor the brickfields under the respective authorities to favor the formation and regeneration of natural vegetation in their neighboring areas.
\end{abstract}

Key words: Floristic composition, Brickfields, Savar-Dhamrai.

\section{INTRODUCTION}

Biodiversity provides all basic ecosystem services, and many valuable components for sustainable development programs throughout the world. This natural resources is being studied by the scientists in several fields (Haidari, 2013; Radaei \& Amini, 2013; Singh \& Kumar, 2013) for exploring the data from different perspectives with different hypotheses. Higher genetic and species diversity tends to make ecosystems more resistant and resilient to the disturbances and adverse changes, and thus ensures their natural functionality and numerous critical services consistently.

The brickfield areas are one kind of semi-urban to rural disturbed areas of Bangladesh that are surrounded mostly by the vegetation of fallow lands and agricultural fields neighboring to the homesteads.

The brick-making industries in Bangladesh producing about 8.7 billion bricks annually are arbitrarily established in different areas throughout the country. These are the best described as "footloose" industries with seasonal production confined to the six dry

\footnotetext{
* Corresponding author. E-mail: mdsaifuli912@gmail.com
} 
months of the year, outdated technology, low labor productivity, non-existent capitalization, and informal management.

The effluents from the brickfields might affect the biodiversity and the air quality of their surrounding and neighboring areas. Because, the respiration and photosynthesis processes in the plants might be affected by the deposition of heat, soot, and smoke particles released from these brickfields on their leaves (Alam et al., 2006; Jensen \& Peppard, 2004; Le \& Oanh, 2010). Additionally, the soil pit excavation makes agricultural areas more vulnerable to the erosive floods which may prevent cultivated land to be enriched by sediment deposition (Alam et al., 2006), and as the consequences, the soil surface might be transformed irreversibly (Singh \& Asgher, 2005). On the other hand, the use of substantial amounts of fuel wood in brick-making industries accelerates deforestation and leads to intensify the emission of Greenhouse Gases (GHGs), such as Carbon dioxide $\left(\mathrm{CO}_{2}\right)$, Carbon monoxide $(\mathrm{CO})$, Methane $\left(\mathrm{CH}_{4}\right)$, Nitrous oxide $\left(\mathrm{N}_{2} \mathrm{O}\right)$, and Nitric oxide (NO; Nitrogen oxide) (Alam \& Starr, 2008). Brick kilns are also associated with $\mathrm{SO}_{2}$, volatile organic compounds, and heavy metals depending on the type of fuel burnt. The contribution of brick kiln emissions to the ambient air quality is estimated from the source apportionment studies (Begum et al., 2006, 2008, 2011) that estimated an average contribution of $30-40 \%$ originating from brick kilns to total ambient PM 2.5 pollution. Brick kiln emissions form a major source of pollution measured over the Dhaka Metropolitan Area (DMA). It has been estimated that at least $35 \%$ of ambient PM10 and at least $15 \%$ of the ambient PM 2.5 in DMA is associated with brick kiln emissions (Azkar et al., 2012). Though numerous brickfields are functional in different areas of Bangladesh, it is yet unknown whether the operation of the brickfields has any impact on the composition and diversity of the plant species growing in their surroundings or neighboring areas, and in what extent they affect this valuable natural resource.

The importance of studying local floristic diversity has been realized and carried out in Bangladesh by many researchers (Khan et al., 1994; Rahman \& Hassan, 1995; Uddin \& Rahman, 1999; Khan \& Huq, 2001; Uddin \& Hassan, 2010). Some of the floristic studies have been conducted on the plain land areas that harbor the brickfields too (Rahman \& Hassan, 1995; Alam et al., 2006; Rahman et al., 2012; Rahman 2013; Rahman et al., 2013; Sarker et al., 2013; Khanam et al., 2020; Roy \& Khan, 2020). However, there is a lack of data on the floristic composition in and around the brickfield areas in comparison to that of other plain land areas in Bangladesh. Therefore, this study was conducted to know the variation in floristic composition with regard to taxonomic enumeration and species diversity of vascular plants in and around the functional and abandoned brickfield areas of Savar-Dhamrai region.

\section{MATERIALS AND METHODS}

The study area belongs to Savar and Dhamrai upazilas of Dhaka district. Savar upazila, with the elevation of 03-20 meter, is located in $23^{\circ} 44^{\prime} 45.1^{\prime \prime}-24^{\circ} 01^{\prime} 56.5^{\prime \prime} \mathrm{N}$ and $90^{\circ} 11^{\prime} 11.4^{\prime \prime}-90^{\circ} 21^{\prime} 18.8^{\prime \prime}$ and Dhamrai upazila, with the elevation of 04-15 meter, is situated in $23^{\circ} 49^{\prime} 42.5^{\prime \prime}-24^{\circ} 02^{\prime} 38.6^{\prime \prime} \mathrm{N}$ and $90^{\circ} 00^{\prime} 18.1^{\prime \prime}-90^{\circ} 14^{\prime} 45.4^{\prime \prime} \mathrm{E}$ (https://elevation. 
maplogs.com; www.google.com/maps; http://haka.gov.bd). Though, the topography of Savar-Dhamrai region is plain to irregularly elevated and the height of land gradually increases from the east to the west and south to the north, the sampling sites of this study, composed of about 380 hectares of land, were confined to the mostly homogenous plain and fallow land areas of the region harboring different functional and abandoned brickfields (Fig. 1).

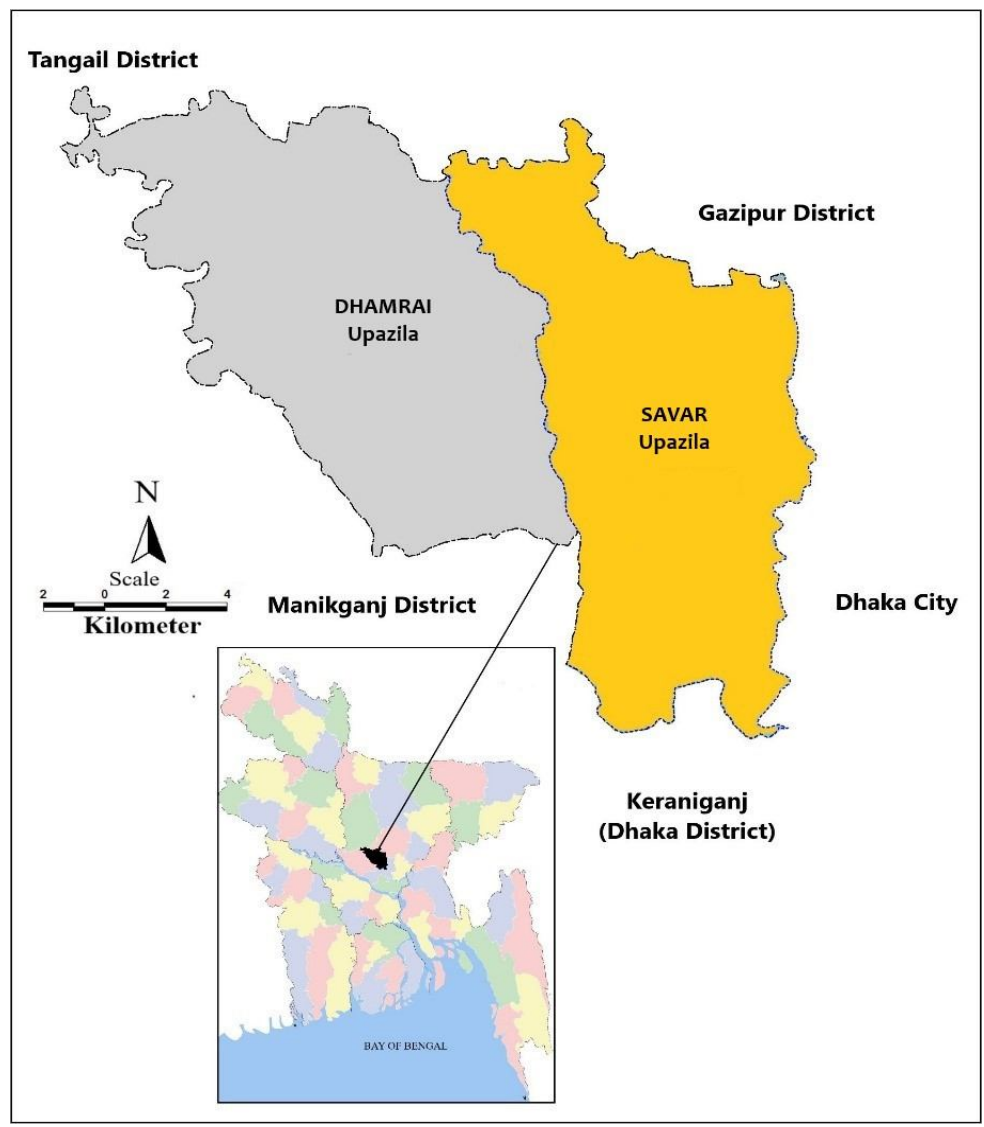

Fig.1. Map of the study area

The land of Savar- and Dhamrai region is composed of alluvium soil of the Pleistocene period (http://dhaka.gov.bd). The main rivers crossing this region are Dhaleshwari, Bangshi, Turag and Karnatali, and other rivers include Kolmai, Gazikhali, and Buriganga. These rivers are mostly polluted by industrial and sewerage wastes and residues of insecticides or herbicides etc. The soil of this region consists of heterogeneous assortment of silt, sand, clay, and gravel and often contains a good deal of organic matter due to which the land of the area is very fertile. The crest soils are represented by brownish grey, dark grey- and grey fine sandy loam soil. Consistency of dry soil is hard, 
moist soil is slightly firm to friable, while nature of wet soil is slightly sticky or plastic (Hossain et al., 2003). This region has a hot, wet and humid tropical climate, monthly humidity varies from $39 \%$ in January-February up to $92 \%$ in July-August, temperature ranges from $13^{\circ} \mathrm{C}$ in January-December to $39^{\circ} \mathrm{C}$ in April-May and total rainfall from 0.6 $\mathrm{mm}$ in November-December to $350.80 \mathrm{~mm}$ in June-July (www.worldweatheronline.com).

Specimens Collection and Preservation: About five hundred fresh specimens representing different taxa were collected from various habitats through the field trips conducted throughout the study area covering different seasons. The specimens were collected only from the healthy twigs with bud, flowers and fruit. The collected specimens were dried and processed using standard herbarium techniques (Bridson \& Forman, 1989; Singh \& Subramaniam, 2008). The voucher specimen of each taxon was selected considering the best representation of the vegetative and reproductive characters. All relevant field information, viz. collection date, locality, habit, habitat, stem height, size, shape, color and odor of the flowers and/or fruits, phenology, distribution, and uses etc. were recorded in the notebook. The dried and mounted specimens are deposited in Jahangirnagar University Herbarium (JUH).

In order to assess the existing plant species diversity in different brickfield areas, quantitative field data were collected through quadrat sampling following the standard quadrat method (Braun-Blanquet, 1932; Raunkiaer, 1934; Cottam et al., 1953; Cottam \& Curtis, 1956; Krebs, 1989). The standard size $\left(1 \mathrm{~m}^{2}\right)$ of the quadrat was determined following the 'Species Area Curve' (Cain, 1938; Braun-Blanquet, 1964).

Specimens Examination and Identification: All plant specimens were preliminarily identified through consulting the experts and matching with relevant voucher specimens preserved at Jahangirnagar University Herbarium (JUH). Taxonomic identification of the specimens were confirmed through matching them with the relevant type images available in the websites of different international herbaria $(K, P, G)$, voucher specimens housed in Bangladesh National Herbarium (DACB), and respective taxonomic descriptions and keys available in standard taxonomic literature (Hooker, 1872-1897; Prain, 1903; Nasir \& Ali, 1980-2005; Wu et al., 1999-2013; Siddiqui et al., 2007; Smitinand \& Larsen, 1975, 1989; Ahmed et al., 2008-2009; Watson et al., 2011; Flora of North America Editorial Committee, 1993-2014). Information on different taxa and their distribution in Bangladesh were verified following Khan (1972-1987), Khan \& Halim (1987), Siddiqui et al. (2007) and Ahmed et al., (2008-2009).

Nomenclatural Information Collection: The updated nomenclatural information of all identified taxa was verified through consulting recent taxonomic publications (Nasir \& Ali, 1980-2005; Wu et al., 1999-2013; Watson et al., 2011) and nomenclatural databases (IPNI, 2019; The Plant List, 2013; TROPICOS, 2020). The local name/s of plant species were based on Huq (1986), Pasha \& Uddin (2013) and interviews with the local people. The angiosperm families are arranged following the classification system of Cronquist (1981), whereas the families of pteridophytes according to the classification system of Pichi (1977). All genera and species are arranged alphabetically. 
Data Analyses: Species diversity was calculated using two diversity indices, viz. Shannon's index (Shannon, 1948) and Simpson's index (Simpson, 1949), using the following formulae:

Shannon's diversity index

$$
H^{\prime}=-\sum p_{i} \ln \left(p_{i}\right)
$$

Where, $H^{\prime}=$ the Shannon diversity index

$\mathrm{pi}=$ the total number of individuals of a particular species/ the total number of individuals of all species

$\ln =\log$ base $n$

$\Sigma=$ sum from species 1 to species $\mathrm{S}$ (numbers of species encountered).

Simpson's diversity index $\mathrm{D}=1-\Sigma \mathrm{n}(\mathrm{n}-1) / \mathrm{N}(\mathrm{N}-1)$

Where, $\mathrm{D}=$ the Simpson diversity index; value ranges between 0 and 1,0 being no diversity and 1 represents infinite diversity

$\mathrm{N}=$ the total number of individuals of all species

$\mathrm{n}=$ the total number of individuals of a particular species

$\Sigma=$ sum from species 1 to species $\mathrm{S}$ (numbers of species encountered).

The similarity between the plant species composition of abandoned and functional brickfield areas was estimated by Jaccard coefficient index using the following formula (Jaccard, 1912; Glen, 2016):

$$
\text { Jaccard Index }=\begin{gathered}
\text { The number of species shared by both sites } \\
\text { Total number of species present in both sites }
\end{gathered} \times 100
$$

\section{RESULTS AND DISCUSSION}

Floristic composition: A total of 181 species, belonging to 133 genera and 51 families, were found in and around the brickfields of Savar-Dhamrai region of Dhaka district. A total 148 species $(81.77 \%)$ were recorded from Savar area and 161 species $(88.95 \%)$ from Dhamrai area, whereas total 128 species $(70.72 \%)$ were commonly shared by these two areas. In the brickfields of Savar-Dhamrai region, the pteridophytes were represented by six species belonging to six genera and five families, the dicotyledons were composed of 128 species belonging to 96 genera and 40 families and the monocotyledons were consisted of 47 species belonging to 31 genera and six families. Among these, the herbs comprised 161 species $(88.95 \%)$ that were followed by shurbs of 14 species $(7.73 \%)$ and trees of six species $(3.32 \%)$ (Table 1$)$. 
During this study, a total of 125 species $(84.46 \%)$ were recorded from the abandoned and 75 species $(50.67 \%)$ from the functional brickfields of Savar area, while 52 species (35.13\%) were commonly shared by these two types of brickfields. In Dhamrai area, a total of $136(84.47 \%)$ species were appeared in the abandoned brickfields and only 80 species $(49.69 \%)$ in the functional brickfields, whereas $55(34.16 \%)$ species were common in abandoned and functional brickfields of this area. A total of 42 species were common in and around the abandoned- and functional brickfield areas of Savar-Dhamrai region. 11 species exclusively occurred in the abandoned brickfields of Savar and 19 in those of Dhamrai, whereas six species in the functional brickfields of both Savar and Dhamrai areas.

These data indicate that the number of vascular plant species in and around the abandoned brickfields of Savar-Dhamrai region was much higher than that in and around the functional brickfields of this region and the brickfields of Dhamrai area harbored somewhat higher number of vascular plant species in respect to the brickfields of Savar area.

Table 1. List of Vascular Plants of brickfield areas of Savar-Dhamrai region of Dhaka district of Bangladesh

\begin{tabular}{|c|c|c|c|c|c|}
\hline Scientific name & Bangla name & Habit & \begin{tabular}{|l|} 
Occurrence \\
\end{tabular} & Uses & Brickfield \\
\hline \multicolumn{6}{|l|}{$\begin{array}{l}\text { MAGNOLIOPSIDA Brongn. } \\
\text { PIPERACEAE Giseke }\end{array}$} \\
\hline Peperomia pellucida (L.) Kunth & Pithapata & Herb, e & $\mathrm{O}$ & M & All \\
\hline MENISPERMACEAE Juss. & & & & & \\
\hline $\begin{array}{l}\text { Stephania japonica (Thunb.) Miers } \\
\text { ULMACEAE Mirb. }\end{array}$ & Akandi manik & Herb, cl & $\mathrm{C}$ & M & $\mathrm{AbD}, \mathrm{FuD}, \mathrm{AbS}$ \\
\hline $\begin{array}{l}\text { Trema orientalis (L.) Blume } \\
\text { MORACEAE Gaudich. }\end{array}$ & Jiban, Banjiga & Tree, $\mathrm{m}$ & $\mathrm{O}$ & Fw, Fd & $\mathrm{FuD}, \mathrm{AbS}$ \\
\hline Ficus heterophylla L.f. & Bhui dumur & Shrub & $\mathrm{C}$ & W & $\mathrm{AbD}$ \\
\hline F. hispida L.f. & Kak dumur & Shrub & $\mathrm{R}$ & $\mathrm{M}$ & All \\
\hline $\begin{array}{l}\text { Streblus asper Lour. } \\
\text { URTICACEAE Juss. }\end{array}$ & Sheora & Tree, $\mathrm{s}$ & $\mathrm{C}$ & M & $\mathrm{AbD}, \mathrm{AbS}, \mathrm{FuS}$ \\
\hline Pilea microphylla (L.) Liebm. & Latamaricha & Herb, pr & $\mathrm{C}$ & $\mathrm{M}$ & All \\
\hline $\begin{array}{l}\text { Pouzolzia zeylanica (L.) Benn. } \\
\text { AMARANTHACEAE Juss. }\end{array}$ & Kullaruki & Herb, pr & $\mathrm{C}$ & M & $\mathrm{AbD}, \mathrm{FuD}$ \\
\hline Achyranthes aspera $\mathrm{L}$. & Apang & Herb, e & $\mathrm{R}$ & M & $\mathrm{AbD}, \mathrm{AbS}$ \\
\hline $\begin{array}{l}\text { Alternanthera paronychioides A. St.- } \\
\text { Hil. }\end{array}$ & Jhuli khata & Herb, pr & $\mathrm{R}$ & M & FuD, FuS \\
\hline A. philoxeroides (Mart.) Griseb. & Henchi & Herb, aq & $\mathrm{C}$ & V & All \\
\hline A. sessilis (L.) R.Br. ex DC. & Malancha & Herb, aq & $\mathrm{C}$ & V & $\mathrm{AbD}, \mathrm{FuD}, \mathrm{AbS}$ \\
\hline Amaranthus blitum L. & Goburanotey & Herb, pr & $\mathrm{C}$ & & FuD \\
\hline A. spinosus $\mathrm{L}$. & Kantanotey & Herb, e & $\mathrm{C}$ & $\mathrm{V}, \mathrm{M}$ & All \\
\hline A. tricolor $L$. & Lalshak & Herb, e & $\mathrm{O}$ & V & $\mathrm{AbD}, \mathrm{AbS}$ \\
\hline A. viridis $\mathrm{L}$. & Notey shak & Herb, e & $\mathrm{C}$ & V & $\mathrm{AbD}$ \\
\hline $\begin{array}{l}\text { Cyathula prostrata (L.) Blume } \\
\text { PORTULACACEAE Juss. }\end{array}$ & Shyontula & Herb, pr & $\mathrm{C}$ & M & AbS \\
\hline $\begin{array}{l}\text { Portulaca oleracea } \mathrm{L} \text {. } \\
\text { BASELLACEAE Raf. }\end{array}$ & Boronunia & Herb, pr & $\mathrm{R}$ & M & All \\
\hline $\begin{array}{l}\text { Basella alba L. } \\
\text { MOLLUGINACEAE Bartl. }\end{array}$ & Pui shak & Herb, cr & $\mathrm{C}$ & V & $\mathrm{AbD}, \mathrm{AbS}$ \\
\hline Glinus oppositifolius (L.) Aug. DC. & Gima Shak & Herb, pr & $\mathrm{O}$ & M & All \\
\hline
\end{tabular}




\begin{tabular}{|c|c|c|c|c|c|}
\hline Scientific name & Bangla name & Habit & Occurrence & Uses & Brickfield \\
\hline Mollugo pentaphylla $\mathrm{L}$. & Khetpapra & Herb, pr & $\mathrm{O}$ & $\mathrm{M}, \mathrm{V}$ & $\mathrm{AbD}, \mathrm{FuS}$ \\
\hline $\begin{array}{l}\text { CARYOPHYLLACEAE Juss. } \\
\text { Polycarpon prostratum (Forssk.) }\end{array}$ & Ghima & Herb, pr & $\mathrm{O}$ & $\mathrm{M}$ & FuD, FuS \\
\hline \multirow{2}{*}{\multicolumn{6}{|c|}{$\begin{array}{l}\text { Asch. \& Schweinf. } \\
\text { POLYGONACEAE Juss. }\end{array}$}} \\
\hline & & & & & \\
\hline Persicaria hydropiper (L.) Delarbre & Biskatali & Herb,e & $\mathrm{O}$ & M & All \\
\hline P. lapathefolia (L.) Delarbre & Pani-bishkatali & Herb, e & $\mathrm{C}$ & M & $\mathrm{AbD}, \mathrm{AbS}$ \\
\hline P. minor (Huds.) Opiz & $\begin{array}{l}\text { Chhoto- } \\
\text { bishkatali }\end{array}$ & Herb, e & $\mathrm{O}$ & - & $\mathrm{AbD}, \mathrm{FuD}$ \\
\hline P.orientalis (L.) Spach & Bara panimarich & Herb, e & $\mathrm{C}$ & - & $\mathrm{AbD}, \mathrm{AbS}$ \\
\hline Polygonum effusum Meisn. & Raniphul & Herb, e & $\mathrm{O}$ & M & $\mathrm{AbD}, \mathrm{AbS}$ \\
\hline P. plebeium $\mathrm{R} . \mathrm{Br}$. & $\begin{array}{l}\text { Khudi- } \\
\text { bishkatali }\end{array}$ & Herb, e & $\mathrm{O}$ & $\mathrm{O}$ & $\mathrm{AbD}, \mathrm{FuD}$ \\
\hline P. praetermissum Hook. f. & Fota bishkatali & Herb, e & $\mathrm{O}$ & - & $\mathrm{AbD}, \mathrm{AbS}$ \\
\hline Rumex dentatus L. & Bon-palang & Herb, e & $\mathrm{C}$ & M & $\mathrm{AbD}, \mathrm{AbS}$ \\
\hline $\begin{array}{l}\text { R. maritimus L. } \\
\text { TILIACEAE Juss. }\end{array}$ & Dati-palang & Herb, e & $\mathrm{C}$ & M & $\mathrm{AbD}, \mathrm{AbS}$ \\
\hline $\begin{array}{l}\text { Triumfetta rhomboidea Jacq. } \\
\text { STERCULIACEAEVent. }\end{array}$ & Bon Okra & Herb, e & $\mathrm{C}$ & M & All \\
\hline $\begin{array}{l}\text { Melochia corchorifolia } \mathrm{L} \text {. } \\
\text { MALVACEAE Juss. }\end{array}$ & Tiki-okra & Herb, e & $\mathrm{O}$ & M & $\mathrm{AbD}, \mathrm{FuD}$ \\
\hline Corchorus aestuans L. & Janglipat & Herb, e & $\mathrm{O}$ & V & $\mathrm{AbD}, \mathrm{AbS}$ \\
\hline Urena lobata $\mathrm{L}$. & Banghagra & Shrub & $\mathrm{C}$ & M & All \\
\hline $\begin{array}{l}\text { Sida rhombifolia } \mathrm{L} \text {. } \\
\text { CUCURBITACEAE Juss. }\end{array}$ & Lal berela & Herb, e & $\mathrm{C}$ & - & All \\
\hline Coccinia grandis (L.) Voigt & Telakucha & Herb, cl & $\mathrm{C}$ & M & $\mathrm{AbD}, \mathrm{AbS}$ \\
\hline Cucumis sativus L. & Khira & Herb, cr & $\mathrm{R}$ & V & $\mathrm{AbD}$ \\
\hline Luffa cylindrica (L.) M.Roem. & Dhundal & Herb, cl & $\mathrm{C}$ & $\mathrm{V}$ & $\mathrm{AbD}, \mathrm{AbS}$ \\
\hline Mukia maderaspatana (L.) M.Roem. & Bilari & Herb, cl & $\mathrm{C}$ & - & $\mathrm{AbD}, \mathrm{AbS}$ \\
\hline $\begin{array}{l}\text { Trichosanthes tricuspidata Lour. } \\
\text { BRASSICACEAE Burnett }\end{array}$ & Makal & Herb, cl & $\mathrm{O}$ & M & $\mathrm{AbD}$ \\
\hline $\begin{array}{l}\text { Rorippa indica (L.) Hiern } \\
\text { CLEOMACEAE Bercht. \& J. Presl }\end{array}$ & Bansarisha & Herb, e & $\mathrm{O}$ & V & All \\
\hline Cleome rutidosperma $\mathrm{DC}$. & Begunehurhurey & Herb, e & $\mathrm{C}$ & $\mathrm{W}$ & $\mathrm{AbD}, \mathrm{AbS}$ \\
\hline C. viscosa $\mathrm{L}$. & Atha hurhuria & Herb, e & $\mathrm{R}$ & $\mathrm{W}$ & $\mathrm{AbD}$ \\
\hline MIMOSACEAE R.Br. & & & & & \\
\hline Acacia auriculiformis Benth. & Akashmoni & Tree, 1 & $\mathrm{C}$ & $\mathrm{T}$ & All \\
\hline Mimosa pudica L. & Lajjaboti & Shrub & $\mathrm{O}$ & M & All \\
\hline CAESALPINIACEAE R.Br. & & & & & \\
\hline Senna occidentalis (L.) Link & Barakalkesunda & Shrub & $\mathrm{C}$ & M & $\mathrm{AbD}, \mathrm{AbS}$ \\
\hline S. sophera (L.) Roxb. & Kalkeshunda & Herb, e & $\mathrm{O}$ & M & $\mathrm{AbD}, \mathrm{FuD}$ \\
\hline S. tora (L.) Roxb. & Terasena & Herb, e & $\mathrm{C}$ & M & All \\
\hline FABACEAE Lindl. & & & & & \\
\hline Aeschynomene indica $\mathrm{L}$. & Bhatshola & Herb, e & $\mathrm{O}$ & $\mathrm{Gm}$ & $\mathrm{AbD}, \mathrm{AbS}$ \\
\hline Cajanus cajan (L.) Millsp. & Arhar & Shrub & $\mathrm{O}$ & $\mathrm{Pu}, \mathrm{Gm}$ & $\mathrm{AbD}$ \\
\hline Desmodium triflorum (L.) DC. & Kulalia & Herb, pr & $\mathrm{C}$ & M & All \\
\hline Sesbania cannabina (Retz.) Pers. & Dhonchi & Herb, e & $\mathrm{R}$ & $\mathrm{Gm}$ & $\mathrm{AbD}$ \\
\hline Tephrosia candida (Roxb.) DC. & Bilakshani & Herb, e & $\mathrm{R}$ & $\mathrm{M}, \mathrm{Gm}$ & $\mathrm{AbS}$ \\
\hline Vicia hirsuta (L.) Gray & Masurechana & Herb, cl & $\mathrm{O}$ & $\mathrm{Fd}$ & $\mathrm{AbD}, \mathrm{AbS}$ \\
\hline Vigna mungo (L.) Hepper & Maskalay & Herb, e & $\mathrm{O}$ & $\mathrm{Pu}, \mathrm{Fd}$ & $\mathrm{AbD}$ \\
\hline LYTHRACEAE J. St.-Hil. & & & & & \\
\hline Rotala rotundifolia (Buch.-Ham. ex & Dim ghurni & Herb, cr & $\mathrm{C}$ & - & $\mathrm{AbD}, \mathrm{AbS}$ \\
\hline
\end{tabular}

Roxb.) Koehne

ONAGRACEAE Juss. 


\begin{tabular}{|c|c|c|c|c|c|}
\hline Scientific name & Bangla name & Habit & \begin{tabular}{|l|} 
Occurrence \\
\end{tabular} & Uses & Brickfield \\
\hline Ludwigia adscendens (L.) H. Hara & Keshordam & Herb, cr & $\mathrm{O}$ & $\mathrm{W}$ & $\mathrm{AbD}, \mathrm{AbS}$ \\
\hline L. hyssopifolia (G. Don) Exell & Panipalong & Herb, e & $\mathrm{O}$ & $\mathrm{W}$ & $\mathrm{AbD}, \mathrm{FuS}$ \\
\hline L. perennis $\mathrm{L}$. & Amorkura & Herb, e & $\mathrm{C}$ & $\mathrm{W}$ & $\mathrm{AbD}, \mathrm{AbS}$ \\
\hline $\begin{array}{l}\text { MAZACEAE Reveal } \\
\text { Mazus pumilus (Burm. f.) Steenis } \\
\text { EUPHORBIACEAE Juss. }\end{array}$ & Tutra & Herb, pr & $\mathrm{C}$ & - & $\mathrm{AbD}, \mathrm{FuD}, \mathrm{AbS}$ \\
\hline Croton bonplandianus Baill. & Banmarich & Herb, e & $\mathrm{C}$ & M & All \\
\hline Euphorbia hirta L. & Bara dudhia & Herb, pr & $\mathrm{C}$ & M & All \\
\hline E. thymifolia L. & Dudhia & Herb, pr & $\mathrm{C}$ & & FuD \\
\hline Phyllanthus niruri L. & Vuiamla & Herb, e & $\mathrm{C}$ & M & All \\
\hline P. reticulatus Poir. & Chitki & Shrub & $\mathrm{O}$ & M & FuS \\
\hline P. urinaria $\mathrm{L}$. & Hazarmani & Herb, e & $\mathrm{O}$ & M & FuD, FuS \\
\hline $\begin{array}{l}\text { Ricinus communis L. } \\
\text { RHAMNACEAE Juss. }\end{array}$ & Bherenda & Shrub & $\mathrm{O}$ & $\mathrm{M}, \mathrm{Ol}$ & $\mathrm{AbD}, \mathrm{AbS}$ \\
\hline $\begin{array}{l}\text { Ziziphus mauritiana Lam. } \\
\text { OXALIDACEAE R. Br. }\end{array}$ & Bol boroi & Tree, $\mathrm{s}$ & $\mathrm{O}$ & M & $\mathrm{AbD}, \mathrm{FuD}$ \\
\hline $\begin{array}{l}\text { Oxalis corniculata } \mathrm{L} \text {. } \\
\text { APIACEAE Lindl. }\end{array}$ & Amrul & Herb, cr & $\mathrm{C}$ & $\mathrm{V}, \mathrm{M}$ & $\mathrm{AbD}, \mathrm{AbS}$ \\
\hline Centella asiatica (L.) Urb. & Thankuni & Herb, cr & $\mathrm{O}$ & M & $\mathrm{AbD}, \mathrm{AbS}$ \\
\hline $\begin{array}{l}\text { Oenanthe benghalensis Benth. \& } \\
\text { Hook. f. } \\
\text { MELIACEAE Juss. }\end{array}$ & Bon-dhonia & Herb, e & $\mathrm{C}$ & - & $\mathrm{AbD}$ \\
\hline $\begin{array}{l}\text { Toona ciliata M. Roem. } \\
\text { SOLANACEAE Juss. }\end{array}$ & Toon & Tree, 1 & $\mathrm{O}$ & $\mathrm{T}$ & $\mathrm{AbD}$ \\
\hline Datura stramonium L. & Sada dhutra & Shrub & $\mathrm{R}$ & M & $\mathrm{AbD}$ \\
\hline $\begin{array}{l}\text { Physalis angulata } \mathrm{L} . \\
\text { Solanum americanum Mill. }\end{array}$ & $\begin{array}{l}\text { Futka } \\
\text { Tit-begun }\end{array}$ & $\begin{array}{l}\text { Herb, e } \\
\text { Herb, e }\end{array}$ & $\begin{array}{l}\mathrm{C} \\
\mathrm{C}\end{array}$ & $\bar{M}$ & $\begin{array}{c}\text { All } \\
\text { FuD, FuS }\end{array}$ \\
\hline S. melongena $\mathrm{L}$. & Begun & Herb, e & $\mathrm{O}$ & V & $\mathrm{FuD}, \mathrm{FuS}$ \\
\hline S. surattense Burm. f. & - & Herb, e & $\mathrm{R}$ & - & $\mathrm{AbS}$ \\
\hline S. torvum $\mathrm{Sw}$. & Gota begun & Herb, e & $\mathrm{C}$ & - & FuD, FuS \\
\hline $\begin{array}{l}\text { Nicotiana plumbaginifolia Viv. } \\
\text { CONVOL VULACEAE Juss. }\end{array}$ & Ban tamak & Herb, e & $\mathrm{O}$ & - & $\mathrm{AbD}, \mathrm{AbS}$ \\
\hline Evolvulus nummularius (L.) L. & Bhui okra & Herb, cr & $\mathrm{C}$ & - & All \\
\hline Ipomoea aquatica Forssk. & Kalmishak & Herb, pr & $\mathrm{C}$ & V & $\mathrm{AbD}, \mathrm{AbS}$ \\
\hline $\begin{array}{l}\text { I. carnea Jacq. } \\
\text { MENYANTHACEAE Dumort. }\end{array}$ & Dhol Kolmi & Shrub & $\mathrm{O}$ & - & $\mathrm{AbD}, \mathrm{AbS}$ \\
\hline $\begin{array}{l}\text { Nymphoides hydrophylla (Lour.) } \\
\text { Kuntze }\end{array}$ & Chand mala & Herb, aq & $\mathrm{C}$ & $\mathrm{W}$ & $\mathrm{AbD}, \mathrm{AbS}$ \\
\hline $\begin{array}{l}\text { N. indica (L.) Kuntze } \\
\text { BORAGINACEAE Juss. }\end{array}$ & Panchuli mala & Herb, aq & $\mathrm{C}$ & W & $\mathrm{AbD}, \mathrm{AbS}$ \\
\hline $\begin{array}{l}\text { Heliotropium indicum } \text { L. } \\
\text { VERBENACEAE J. St.-Hil. }\end{array}$ & Hatisur & Herb, e & $\mathrm{C}$ & M & $\mathrm{AbD}, \mathrm{FuD}, \mathrm{AbS}$ \\
\hline Phyla nodiflora (L.) Greene & Vuiokra & Herb, cr & $\mathrm{C}$ & M & $\mathrm{AbS}, \mathrm{FuS}$ \\
\hline Lantana camara $\mathrm{L}$. & Kutuskanta & Shrub & $\mathrm{R}$ & M & $\mathrm{AbD}, \mathrm{AbS}$ \\
\hline $\begin{array}{l}\text { Lippia alba (Mill.) N.E.Br. ex Britton } \\
\text { \& P.Wilson } \\
\text { LAMIACEAE Martinov }\end{array}$ & Pichas-lakri & Shrub & $\mathrm{C}$ & & FuD, FuS \\
\hline Clerodendrum infortunatum $\mathrm{L}$. & Bhat & Shrub & $\mathrm{C}$ & M & $\mathrm{AbD}, \mathrm{AbS}$ \\
\hline Hyptis capitata Jacq. & Tata tokma & Herb, e & $\mathrm{C}$ & $\mathrm{W}$ & FuD, FuS \\
\hline H. suaveolens (L.) Poit. & Tokma & Herb, e & $\mathrm{C}$ & M & $\mathrm{AbD}, \mathrm{AbS}$ \\
\hline Leucas zeylanica (L.) W.T. Aiton & Dondokalosh & Herb, e & $\mathrm{O}$ & M & All \\
\hline Leonurus sibiricus L. & Rokto-dron & Herb, e & $\mathrm{R}$ & $\mathrm{W}$ & FuD \\
\hline Ocimum americanum $\mathrm{L}$. & Bantulsi & Herb, e & $\mathrm{O}$ & M & $\mathrm{AbD}, \mathrm{AbS}, \mathrm{FuS}$ \\
\hline Pogostemon auricularius (L.) Hassk. & Aripachuli & Herb, e & $\mathrm{O}$ & M & AbS \\
\hline
\end{tabular}




\begin{tabular}{|c|c|c|c|c|c|}
\hline Scientific name & Bangla name & Habit & Occurrence & Uses & Brickfield \\
\hline $\begin{array}{l}\text { PLANTAGINACEAE Juss. } \\
\text { Limnophila heterophylla (Roxb.) } \\
\text { Benth. }\end{array}$ & Patakutra & Herb, e & $\mathrm{O}$ & $\mathrm{W}$ & FuD \\
\hline $\begin{array}{l}\text { Mecardonia procumbens (Mill.) } \\
\text { Small }\end{array}$ & Micardan & Herb, cr & $\mathrm{C}$ & $\mathrm{W}$ & $\mathrm{FuD}, \mathrm{AbS}$ \\
\hline $\begin{array}{l}\text { Scoparia dulcis L. } \\
\text { LINDERNIACEAE Borsch, Kai Mi }\end{array}$ & $\begin{array}{l}\text { Bondhone } \\
\text { 11l. \& Eb. Fisch. }\end{array}$ & Herb, e & $\mathrm{O}$ & M & All \\
\hline Lindernia antipoda (L.) Alston & Zai ghas & Herb, pr & $\mathrm{C}$ & $\mathrm{W}$ & $\mathrm{AbD}, \mathrm{AbS}$ \\
\hline L. ciliata (Colsm.) Pennell & Bhui papri & Herb, pr & $\mathrm{C}$ & - & $\mathrm{AbD}, \mathrm{AbS}, \mathrm{FuS}$ \\
\hline L. crustacea (L.) F.Muell. & Chapra ghas & Herb, pr & $\mathrm{C}$ & - & All \\
\hline $\begin{array}{l}\text { L. rotundifolia (L.) Alston } \\
\text { ACANTHACEAE Juss. }\end{array}$ & Tan chapra & Herb, e & $\mathrm{C}$ & - & $\mathrm{AbD}, \mathrm{AbS}$ \\
\hline $\begin{array}{l}\text { Hemigraphis hirta (Vahl) } \mathrm{T} \text {. } \\
\text { Anderson }\end{array}$ & Buripana & Herb, pr & $\mathrm{R}$ & M & All \\
\hline Hygrophila erecta (Burm.f.) Hochr & Filareck & Herb, e & $\mathrm{O}$ & $\mathrm{W}$ & FuD, AbS \\
\hline H. polysperma (Roxb.) T. Anderson & Alai kalai & Herb, pr & $\mathrm{O}$ & $\mathrm{W}$ & $\mathrm{AbD}, \mathrm{AbS}$ \\
\hline Nelsonia canescens (Lam.) Spreng. & Paramul & Herb, e & $\mathrm{C}$ & - & FuS \\
\hline $\begin{array}{l}\text { Rungia pectinata } \text { (L.) Nees } \\
\text { PEDALIACEAER. Br. }\end{array}$ & Pindi & Herb, pr & $\mathrm{O}$ & M & All \\
\hline $\begin{array}{l}\text { Sesamum indicum L. } \\
\text { RUBIACEAE Juss. }\end{array}$ & Til & Herb, e & $\mathrm{O}$ & M & $\mathrm{AbD}, \mathrm{AbS}$ \\
\hline $\begin{array}{l}\text { Catunaregam spinosa (Thunb.) } \\
\text { Tirveng. }\end{array}$ & Mankanta & Shrub & $\mathrm{C}$ & M & $\mathrm{AbS}, \mathrm{FuS}$ \\
\hline $\begin{array}{l}\text { Dentella repens (L.) J.R.Forst. \& } \\
\text { G.Forst. }\end{array}$ & Bhuipat & Herb, pr & $\mathrm{C}$ & - & FuS \\
\hline Oldenlandia corymbosa $\mathrm{L}$. & Khet papra & Herb, pr & $\mathrm{C}$ & - & $\mathrm{AbD}, \mathrm{FuD}$ \\
\hline O. diffusa (Willd.) Roxb. & Fussa papra & Herb, pr & $\mathrm{C}$ & - & $\mathrm{AbD}$ \\
\hline $\begin{array}{l}\text { Richardia scabra L. } \\
\text { ASTERACEAE Bercht. \& J. Presl }\end{array}$ & Nakli ipecac & Herb, e & $\mathrm{R}$ & - & FuD \\
\hline Ageratum conyzoides $(\mathrm{L}.) \mathrm{L}$. & Fulkuri & Herb, e & $\mathrm{C}$ & $\mathrm{M}$ & All \\
\hline Bidens pilosa $\mathrm{L}$. & Bidenlosa & Herb, e & $\mathrm{R}$ & $\mathrm{W}$ & $\mathrm{AbD}$ \\
\hline Blumea lacera (Burm.f.) DC. & Barokukshim & Herb, e & $\mathrm{O}$ & M & $\mathrm{AbD}, \mathrm{AbS}$ \\
\hline Cyanthillium cinereum (L.) H.Rob. & Shialmutra & Herb, e & $\mathrm{O}$ & M & All \\
\hline Eclipta prostrata (L.) L. & Kalokeshi & Herb, pr & $\mathrm{C}$ & M & All \\
\hline Enydra fluctuans DC. & Helencha & Herb, pr & $\mathrm{C}$ & V & FuD, AbS \\
\hline Grangea maderaspatana (L.) Poir. & Namuti & Herb, e & $\mathrm{O}$ & M & $\mathrm{AbD}, \mathrm{FuS}$ \\
\hline Mikania micrantha Kunth & Assamlata & Herb, cl & $\mathrm{C}$ & M & $\mathrm{AbD}, \mathrm{AbS}$ \\
\hline Sonchus arvensis $\mathrm{L}$. & Chashar & Herb, e & $\mathrm{R}$ & $\mathrm{W}$ & $\mathrm{AbD}$ \\
\hline Spilanthes acmella (L.) L. & Mahatitinga & Herb, pr & $\mathrm{C}$ & $\mathrm{O}, \mathrm{M}$ & $\mathrm{AbD}, \mathrm{AbS}, \mathrm{FuS}$ \\
\hline Synedrella nodiflora (L.) Gaertn. & Nakphul & Herb, e & $\mathrm{C}$ & - & All \\
\hline Tridax procumbens (L.) L. & Tridhara & Herb, e & $\mathrm{C}$ & M & $\mathrm{AbD}, \mathrm{FuS}$ \\
\hline Xanthium strumarium $\mathrm{L}$. & Ghagra & Herb, e & $\mathrm{C}$ & - & $\mathrm{AbD}, \mathrm{AbS}$ \\
\hline $\begin{array}{l}\text { Youngia japonica }(\mathrm{L} .) \text { DC. } \\
\text { LILIOPSIDABatsch }\end{array}$ & Youngaful & Herb, e & $\mathrm{R}$ & - & FuD, AbS, FuS \\
\hline $\begin{array}{l}\text { ARECACEAE Bercht. \& J. Presl } \\
\text { Phoenix sylvestris (L.) Roxb. } \\
\text { ARACEAEJuss. }\end{array}$ & Deshi khejur & Tree, 1 & $\mathrm{C}$ & Fw, Ju & $\mathrm{AbD}, \mathrm{AbS}$ \\
\hline Colocasia esculenta (L.) Schott & Jangli kachu & Herb, e & $\mathrm{C}$ & $\mathrm{V}, \mathrm{M}$ & $\mathrm{AbD}, \mathrm{AbS}$ \\
\hline Lemna minor $\mathrm{L}$. & Sujipana & Herb, aq & $\mathrm{C}$ & $\mathrm{Gm}$ & $\mathrm{AbS}$ \\
\hline L. perpusilla Torr. & Khudipana & Herb, aq & $\mathrm{C}$ & $\mathrm{Gm}$ & $\mathrm{AbD}, \mathrm{AbS}$ \\
\hline Pistia stratiotes $\mathrm{L}$. & Topapana & Herb, aq & $\mathrm{C}$ & $\mathrm{Gm}$ & $\mathrm{AbD}, \mathrm{AbS}$ \\
\hline Typhonium flagelliforme (Lodd.) & Ghechu & Herb, e & $\mathrm{C}$ & V & $\mathrm{AbD}, \mathrm{FuS}$ \\
\hline
\end{tabular}

COMMELINACEAEMirb. 


\begin{tabular}{|c|c|c|c|c|c|}
\hline Scientific name & Bangla name & Habit & Occurrence & Uses & Brickfield \\
\hline Commelina benghalensis L. & Kanshira & Herb, e & $\mathrm{O}$ & $\mathrm{M}$ & All \\
\hline C. diffusa Burm.f. & $\begin{array}{l}\text { Monayna } \\
\text { kanshira }\end{array}$ & Herb, e & $\mathrm{C}$ & M & FuD, FuS \\
\hline C. longifolia Lam. & Pani kanshira & Herb, pr & $\mathrm{C}$ & W & AbS \\
\hline $\begin{array}{l}\text { Murdannia nudiflora (L.) Brenan } \\
\text { CYPERACEAE Juss. }\end{array}$ & Kureli & Herb, e & $\mathrm{O}$ & - & FuS \\
\hline Cyperus difformis L. & Behua ghasi & Herb, e & $\mathrm{C}$ & W & All \\
\hline C. haspan $\mathrm{L}$. & Haspan ghasi & Herb, e & $\mathrm{C}$ & W & AbS \\
\hline C. iria L. & Barachucha & Herb, e & $\mathrm{C}$ & $\mathrm{Sb}$ & $\mathrm{FuD}, \mathrm{AbS}$ \\
\hline C. laxus Lam. & Alga ghasi & Herb, e & $\mathrm{C}$ & $\mathrm{Sb}$ & FuD \\
\hline C. rotundus $\mathrm{L}$. & Nagarmutha & Herb, e & $\mathrm{C}$ & $\mathrm{Sb}$ & All \\
\hline $\begin{array}{l}\text { Cyanotis axillaris (L.) D.Don ex } \\
\text { Sweet }\end{array}$ & Axinot & Herb, cr & $\mathrm{C}$ & $\mathrm{W}$ & $\mathrm{AbS}$ \\
\hline $\begin{array}{l}\text { Fimbristylis bisumbellata (Forssk.) } \\
\text { Bubani }\end{array}$ & Bisu fimbry & Herb, e & $\mathrm{C}$ & $\mathrm{W}$ & $\mathrm{FuD}, \mathrm{AbS}$ \\
\hline F. dichotoma (L.) Vahl & Bara nirbishi & Herb, e & $\mathrm{C}$ & $\mathrm{W}$ & All \\
\hline F. quinquangularis (Vahl) Kunth & $\begin{array}{l}\text { Pachkona } \\
\text { fibmry }\end{array}$ & Herb, e & $\mathrm{O}$ & W & $\mathrm{AbD}, \mathrm{AbS}$ \\
\hline F. schoenoides (Retz.) Vahl & Kesari Malanga & Herb, e & $\mathrm{C}$ & - & $\mathrm{AbD}, \mathrm{FuD}, \mathrm{AbS}$ \\
\hline $\begin{array}{l}\text { Kyllinga nemoralis (J.R.Forst. \& } \\
\text { G.Forst.) Dandy ex Hutch. \& Dalziel } \\
\text { POACEAE Barnhart }\end{array}$ & Subashinirbisa & Herb, e & $\mathrm{C}$ & $\mathrm{Fd}$ & $\mathrm{AbD}, \mathrm{AbS}, \mathrm{FuS}$ \\
\hline Apluda mutica $\mathrm{L}$. & Matica & Herb, e & $\mathrm{R}$ & $\mathrm{Fd}$ & AbS \\
\hline $\begin{array}{l}\text { Chrysopogon zizanioides (L.) } \\
\text { Roberty }\end{array}$ & Bena & Herb, e & $\mathrm{O}$ & $\mathrm{Fd}, \mathrm{Sb}$ & $\mathrm{AbD}, \mathrm{FuS}$ \\
\hline Cynodon dactylon (L.) Pers. & Durba ghas & Herb, pr & $\mathrm{C}$ & $\mathrm{Fd}, \mathrm{Sb}$ & All \\
\hline $\begin{array}{l}\text { Dactyloctenium aegyptium (L.) } \\
\text { Willd. }\end{array}$ & Kakpaya & Herb, e & $\mathrm{O}$ & - & FuD, AbS, FuS \\
\hline $\begin{array}{l}\text { Digitaria bicornis (Lam.) Roem. \& } \\
\text { Schult. }\end{array}$ & Baikochira & Herb, e & $\mathrm{C}$ & $\mathrm{Sb}$ & $\mathrm{AbD}$ \\
\hline D. ciliaris (Retz.) Koeler & Kokjachira & Herb, e & $\mathrm{C}$ & - & All \\
\hline D. sanguinalis (L.) Scop. & Mukurjoli & Herb, e & $\mathrm{O}$ & - & All \\
\hline Echinochloa colona (L.) Link. & Shama Ghas & Herb,e & $\mathrm{C}$ & $\mathrm{Fd}$ & $\mathrm{AbD}, \mathrm{FuD}$ \\
\hline Eleusine indica (L.) Gaertn. & Malankuri & Herb, e & $\mathrm{C}$ & $\mathrm{Fd}$ & All \\
\hline $\begin{array}{l}\text { Eragrostis amabilis (L.) Wight \& } \\
\text { Arn. }\end{array}$ & Koni ghas & Herb, e & $\mathrm{O}$ & $\mathrm{Fd}, \mathrm{Sb}$ & $\mathrm{AbD}$ \\
\hline E. cilianensis (All.) Janch. & Dudh-nol & Herb, e & $\mathrm{O}$ & $\mathrm{W}$ & FuS \\
\hline E. unioloides (Retz.) Nees ex Steud. & Chirakoni & Herb, e & $\mathrm{C}$ & $\mathrm{Fd}, \mathrm{Gm}$ & All \\
\hline $\begin{array}{l}\text { Hygroryza aristata (Retz.) Nees ex } \\
\text { Wight \& Arn. }\end{array}$ & Jongli dhan & Herb, e & $\mathrm{O}$ & $\mathrm{W}$ & $\mathrm{AbD}, \mathrm{AbS}$ \\
\hline Imperata cylindrica (L.) Raeusch. & Chhan & Herb, e & $\mathrm{C}$ & $\mathrm{Fd}, \mathrm{M}$ & All \\
\hline Leersia hexandra Sw. & Fulka ghas & Herb, cr & $\mathrm{C}$ & $\mathrm{Fd}$ & $\mathrm{AbS}$ \\
\hline Oryza rufipogon Griff. & Bunodhan & Herb, e & $\mathrm{R}$ & $\mathrm{Fd}$ & $\mathrm{AbD}$ \\
\hline O. sativa $\mathrm{L}$. & Dhan & Herb, e & $\mathrm{O}$ & $\mathrm{Fd}$ & $\mathrm{AbD}$ \\
\hline Ottochloa nodosa (Kunth) Dandy & Voyal ghas & Herb, e & $\mathrm{O}$ & - & AbS \\
\hline Paspalum conjugatum P.J. Bergius. & Moisshya ghas & Herb, e & $\mathrm{C}$ & - & $\mathrm{AbS}, \mathrm{FuS}$ \\
\hline P. scrobiculatum $\mathrm{L}$. & Bishmona ghas & Herb, e & $\mathrm{C}$ & - & FuS \\
\hline Pennisetum glaucum (L.) R.Br. & Kawni & Herb, e & $\mathrm{C}$ & $\mathrm{Fd}$ & $\mathrm{AbD}, \mathrm{AbS}$ \\
\hline $\begin{array}{l}\text { Rottboellia cochinchinensis (Lour.) } \\
\text { Clayton }\end{array}$ & $\begin{array}{l}\text { Boro-sowati } \\
\text { ghas }\end{array}$ & Herb, e & $\mathrm{O}$ & $\mathrm{Fd}$ & $\mathrm{AbD}$ \\
\hline Saccharum spontaneum $\mathrm{L}$. & Kash & Herb, e & $\mathrm{O}$ & Fw & $\mathrm{AbD}, \mathrm{AbS}$ \\
\hline Setaria pumila (Poir.) Roem. \& & Halde kawn & Herb, e & $\mathrm{O}$ & $\mathrm{Fd}$ & $\mathrm{FuD}, \mathrm{FuS}$ \\
\hline
\end{tabular}

Schult.

PONTEDERIACEAE Kunth 


\begin{tabular}{|c|c|c|c|c|c|}
\hline Scientific name & Bangla name & Habit & \begin{tabular}{|l|} 
Occurrence \\
\end{tabular} & Uses & Brickfield \\
\hline Eichhornia crassipes (Mart.) Solms & Kachuripana & Herb, aq & $\mathrm{C}$ & $\mathrm{Gm}$ & $\mathrm{AbD}, \mathrm{AbS}$ \\
\hline $\begin{array}{l}\text { Monochoria hastata (L.) Solms } \\
\text { PTERIDOPHYTA Schimp. }\end{array}$ & Bara nukha & Herb, aq & $\mathrm{C}$ & $\mathrm{Fd}$ & $\mathrm{AbD}, \mathrm{AbS}$ \\
\hline $\begin{array}{l}\text { SALVINIACEAE Martinov } \\
\text { Salvinia cucullata } \text { Roxb. } \\
\text { MARSILEACEAE Mirb. }\end{array}$ & Indur kani & Herb, aq & $\mathrm{C}$ & $\mathrm{Gm}$ & $\mathrm{AbD}, \mathrm{AbS}$ \\
\hline $\begin{array}{l}\text { Marsilea minuta } \mathrm{L} \text {. } \\
\text { LYGODIACEAE M. Roem. }\end{array}$ & Susni shak & Herb, cr & $\mathrm{C}$ & M & All \\
\hline $\begin{array}{l}\text { Lygodium flexuosum (L.) Sw. } \\
\text { PTERIDACEAE E.D.M. Kirchn. }\end{array}$ & Saralata fern & Herb, cr & $\mathrm{C}$ & $\mathrm{W}$ & $\mathrm{AbD}, \mathrm{AbS}$ \\
\hline $\begin{array}{l}\text { Ceratopteris thalictroides (L.) } \\
\text { Brongn. }\end{array}$ & Pani lettuce & Herb, aq & $\mathrm{O}$ & $\mathrm{V}$ & $\mathrm{FuD}, \mathrm{AbS}$ \\
\hline $\begin{array}{l}\text { Pteris vittata } \text { L. } \\
\text { THELYPTERIDACEAE Ching ex } \\
\text { Pic. Serm. }\end{array}$ & Imoditeris & Herb, pr & $\mathrm{C}$ & W & All \\
\hline Ampelopteris prolifera (Retz.) Copel. & $\begin{array}{l}\text { Lombo dheki } \\
\text { shak }\end{array}$ & Herb, cr & $\mathrm{C}$ & V & $\mathrm{AbD}, \mathrm{FuS}$ \\
\hline
\end{tabular}

Note: Habit: $\mathrm{aq}=$ aquatic, $\mathrm{cl}=$ climber, $\mathrm{cr}=$ creeping, $\mathrm{e}=$ erect, $\mathrm{l}=$ large, $\mathrm{m}=$ medium, $\mathrm{pr}=$ prostrate, $\mathrm{s}=$ small. Occurrence: $\mathrm{C}=$ Common, $\mathrm{O}=$ Occasional, $\mathrm{R}=$ Rare. Use: $\mathrm{Fb}=$ fibre, $\mathrm{Fd}=$ fodder, $\mathrm{Fw}=$ fuel wood, $\mathrm{Gm}=$ green manure, $\mathrm{Ju}=$ juice $\mathrm{M}=$ medicine, $\mathrm{O}=$ ornamental, $\mathrm{Pu}=$ pulse, $\mathrm{Ol}=$ oil, $\mathrm{Sb}=$ soil binder, $\mathrm{T}=$ timber, and $\mathrm{V}=$ vegetable, $\mathrm{W}=$ weed. Brickfield Type: $\mathrm{AbD}=$ abandoned brickfield of Dhamrai; FuD = functional brickfield of Dhamrai; $\mathrm{AbS}=$ abandoned brickfield of Savar; FuS = functional brickfield of Savar. $\mathrm{All}=\mathrm{AbD}, \mathrm{FuD}, \mathrm{AbS}$ and FuS.

In the pteridophytes of the studied brickfield areas, Pteridaceae was the largest family with two species only, while Salviniaceae, Marsileaceae, Lygodiaceae and Thelypteridaceae were represented by a single species each.In Magnoliopsida (dicotyledons), Asteraceae with 14 species was the largest family followed by Amaranthaceae, and Polygonaceae with nine species each; while Poaceae with 24 species was the largest family in Liliopsida (monocotyledons), followed by Cyperaceae with 11 species. Amaranthus L. of Amaranthaceae, Lindernia All. of Linderniaceae, Persicaria Mill. of Polygonaceae and Solanum L. of Solanaceae with four species each were the largest genera in Magnoliopsida, whereas, Cyperus L. of Cyperaceae with five species was the largest genus in the Liliopsida. In Magnoliopsida, the genera Alternanthera Forssk., Polygonum L., Ludwigia L., Phyllanthus L. and Senna Mill. were found with three species each; while Ficus L., Rumex L., Cleome L., Euphorbia L., Ipomoea L., Nymphoides Ség., Hyptis Jacq., Hygrophilla R. Br. and Oldenlandia L. with two species each. A total of 47 genera were appeared with single species each. Alternanthera spinosus, Cynodon dactylon, Cyperus rotundus, Eclipta prostrata, Pilea microphylla, and Synedrella nodiflora etc. were common in the brickfields of Savar and Dhamrai region. Additionally, Phyla nodiflora and Paspalum conjugatum were common in the brickfields of Savar area, whereas Pouzolzia zeylanica and Echinochloa colona in those of Dhamrai area.

In Liliopsida, Fimbristylis Vahl. was recorded as the largest genus with four species, followed by Commelina L., Digitaria Haller and Eragrostis Host ex Hitchc. with three species each, Lemna L., Oryza L. and Paspalum L. with two species each and a total of 
23 genera were represented by a single species each. Cynodon dactylon, Eleusine indica, Eragrostis unioloides, Fimbristylis dichotoma and Cyperus rotundus etc. were common in the brickfields of Savar and Dhamrai region. Paspalum conjugatum was documented as the most common species in the brickfields of Savar, whereas Echinochloa colona in the brickfields of Dhamrai area.

During this study, a total of 144 species were recognized as economically important. The major categories of these economically important species were medicinal (67 species), weed (28 species), fodder (20 species), vegetable (19 species), green manure (10 species), soil binder (seven species), fuel wood and oil yielding (three species), and pulse (two species). 16 species were documented as useful in two categories.

The similarity in species composition estimated by Jaccard coefficient shows that the brickfield areas of Savar and Dhamrai were $23.20 \%$ similar in the composition of vascular plant species. The highest similarity (64.15\%) was shared by the abandoned brickfields of Savar and those of Dhamrai which was followed by that shared by the functional brickfields $(53.46 \%)$ of these two areas. The similarity between the functional and abandoned brickfields of Savar area was closer to those of Dhamrai area (Fig. 2).

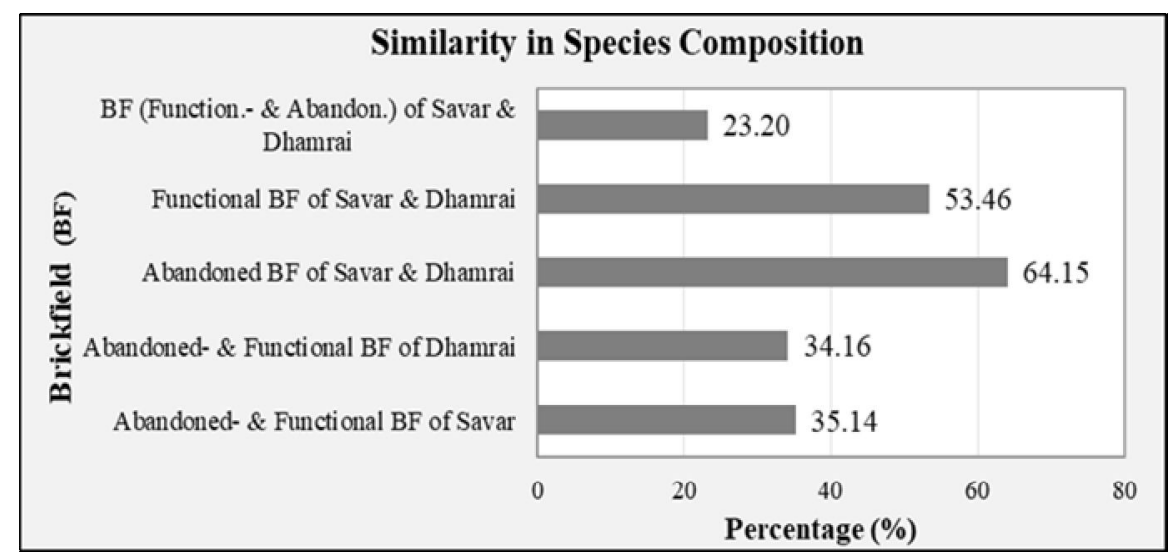

Fig. 2. Similarity between the brickfields of Savar-Dhamrai region in species composition based on Jaccard Coefficient (Jaccard, 1912)

The result shows that the flora of all brickfield areas of Savar and Dhamrai region covered by this study seem to be poor in species composition in respect to that of some forest areas (Arefin et al., 2011) and semi-urban to rural areas (Sultana et al., 2013). The taxonomic enumeration of the species extant in the study area seems somewhat higher than that of few previous records from forest areas (Rashid \& Mia, 2001; Rahman \& Hassan, 1995; Uddin \& Hassan, 2010) and semi-urban or rural areas (Anonymous, 2012a; Anonymous, 2012b; Rahman et al., 2012), if the size of the sampling area is considered. 
Species diversity: The calculated values of Shannon's and Simpson's diversity indices for functional and abandoned brickfields of the study area show that species diversity of the sampling sites was increased in increase of their distance up to certain level (ca. 550 ft) from the brickfields and the abandoned brickfield areas harbored much higher species diversity than that of functional brickfield areas (Figs. 3 and 4).

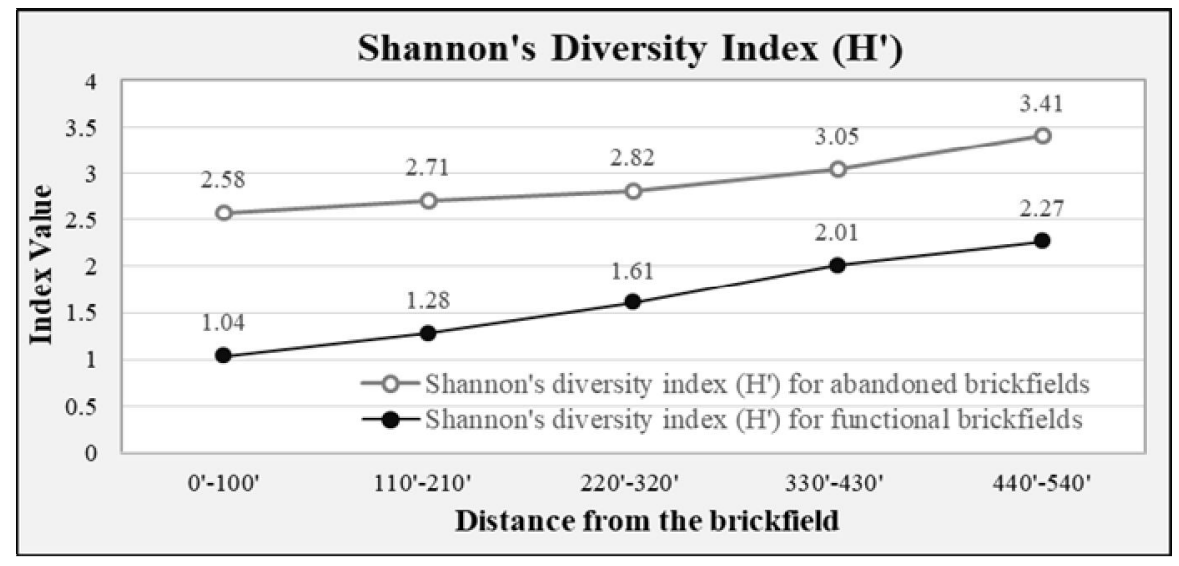

Fig. 3. Comparison between functional and abandoned brickfields of Savar-Dhamrai areas in species diversity of vascular plants based on Shannon's diversity index values

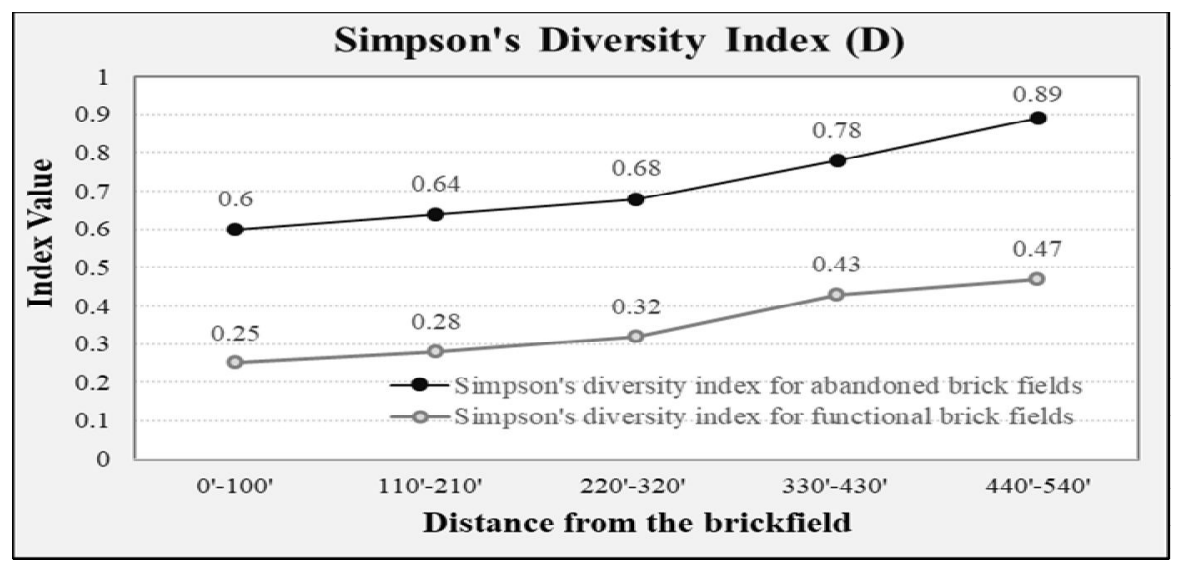

Fig. 4. Comparison between functional and abandoned brickfields of Savar-Dhamrai areas in species diversity of vascular plants based on Simpson's diversity index values 


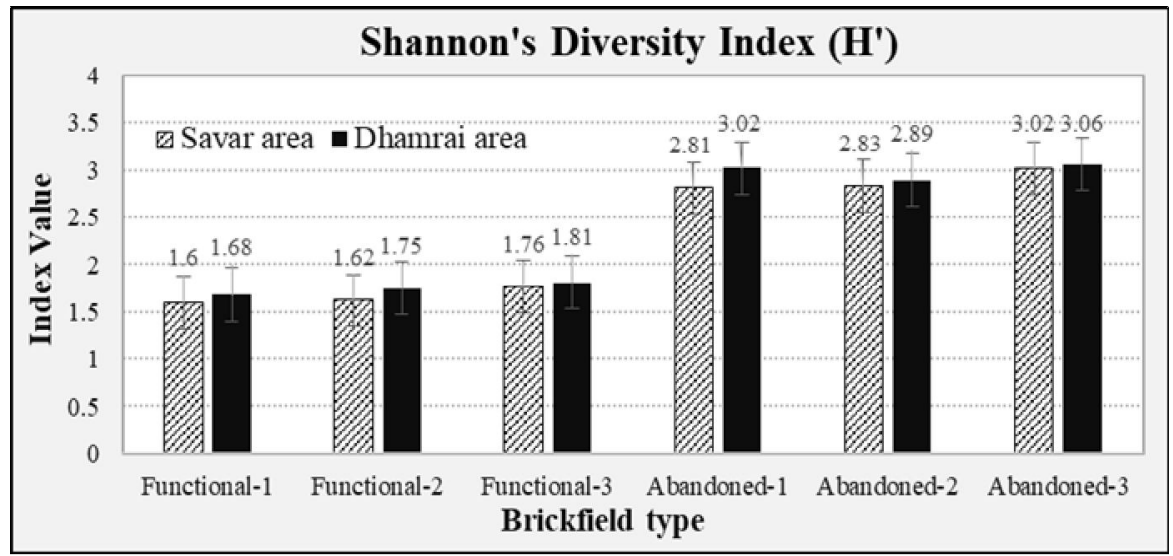

Fig. 5. Variation in Shannon's diversity index values for the vascular plants of functional and abandoned brickfields of three representative sites of each of Savar- and Dhamrai upazilas

The calculation of Shannon index (Fig. 3) shows that the diversity value of the adjacent or nearest area of abandoned brickfields $\left(0^{\prime}-100^{\prime}\right.$ distance) was 2.58 , whereas, for functional field was 1.04. The species diversity of abandoned and functional brickfields at more than $100^{\prime}$ distance were found to be increased up to 3.41 and 2.27 at 440'-540' distance, respectively, and after that the difference in species diversity of these two types of brickfields was gradually decreased. These data indicate that the functional brickfields harbor less species diversity than that of the abandoned brickfields, especially at their adjacent areas, and this trend is reflected in their neighboring areas up to certain distance. The diversity values based on Simpson's index show the same trend as that of Shannon's index in case of increasing species diversity in abandoned brickfield in respect to functional brickfields at all selected distances in the study area (Fig. 4). The Simpson's diversity index value of abandoned and functional fields of Savar and Dhamrai area at 0'$100^{\prime}$ distance is 0.6 and 0.25 , respectively, at $110^{\prime}-210^{\prime}$ distance is 0.64 and 0.28 , at $220^{\prime}-$ $320^{\prime}$ distance is 0.68 and 0.32 , at $330^{\prime}-430^{\prime}$ distance is 0.78 and 0.43 and at $440^{\prime}-540^{\prime}$ distance is 0.89 and 0.47 (Fig. 4).

A comparison between the species diversity of functional and abandoned brickfields of three representative sites of each of Savar- and Dhamrai upazilas based on the average values of Shannon's index (Fig. 5) shows that it is remarkably variable but the functional brickfields harbor lower range of species diversity (1.6-1.81) than that (2.81-3.06) of the abandoned brickfields, and both the functional and abandoned brickfields of Dhamrai area (1.68-1.81 and 2.89-3.06, respectively) harbor somewhat higher species diversity than those of Savar area (1.60-1.76 and 2.81-3.02, respectively), though both of these two upazilas have almost similar type of vegetation. 
The species diversity recorded from the studied brickfield areas of Savar and Dhamrai region is consistent with the reports on few forest areas (Rahman \& Hassan, 1995; Rashid \& Mia, 2001) and semi-urban to rural areas (Alam et al., 2006; Rahman et al., 2012; Shetu et al., 2018; Nahaer, 2014). To know the exact reasons of the occurrence of higher species composition and diversity of vascular plants in and around the brickfield areas of Savar and Dhamrai region than those of some plain land areas of Bangladesh, as reported by some previous studies, further comparative studies involving more parameters are necessary. However, the dense and mostly herbaceous vegetation cover in most of the fallow lands or abandoned plain lands near or adjacent to the brickfield areas of the region, mode and frequency of functional anthropogenic interferences, and the sampling and data collection methods followed in these studies might be recognized as the potential factors. It is also notable that, once in the past, the study area was almost rural and covered with very dense vegetation, though recently the species composition and floristic diversity of the area have been notably decreased due to different anthropogenic activities.

In respect to different threats to the flora and plant diversity of Savar and Dhamrai region comprising a good number of economically important species, this study suggests to control the establishment new brickfields, make sure the operation of the good quality brickfields and monitor these under the respective authorities. As the consequence of rapid urbanization, agricultural expansion, and increasing industrialization etc., the study area might loss its natural vegetation completely in near future. Therefore, this study recommends to manage the abandoned brickfields properly to favor the formation of natural vegetation there that can serve as the micro-ecosystems and tiny spots of biodiversity in this region. Additionally, undertaking enough initiatives to increase public awareness for the conservation of biological resources in this region is also recommended.

\section{REFERENCES}

Ahmed, Z.U., Begum, Z.N.T., Hassan, M.A., Khondker, M., Kabir, S.M.H., Ahmad, M., Ahmed, A.T.A., Rahman, A.K.A. and Haque, E.U. (eds.). 2008-2009. Encyclopedia of Flora and Fauna of Bangladesh. Vols. 6-8, 12. Asiatic Society of Bangladesh, Dhaka.

Ahmed, Z.U., Hassan, M.A., Begum, Z.N.T., Khondker, M., Kabir, S.M.H., Ahmad, M., Ahmed, A.T.A. (eds.). 2009. Encyclopedia of Flora and Fauna of Bangladesh. Vols. 9, 10. Asiatic Society of Bangladesh, Dhaka.

Alam, M.S., Hassan, M.A. and Uddin, M.Z. 2006. A Preliminary Checklist of the angiospermic flora of Ghagotia union under Kapasia upazila in Gazipur district, Bangladesh. Bangladesh J. Plant Taxon. 13(2): 155-170.

Alam, S.A. and Starr, M. 2008. Deforestation and greenhouse gas emissions associated with fuelwood consumption of the brick making industry in Sudan. Science of the Total Environ. 407(2): 847-852.

Anonymous, 2012a. Angiospermic Flora of Lakshmipur Sadar Upazila, Lakshmipur, M.S. Thesis (Unpublished), Plant Taxonomy and Ethnobotany Laboratory, Department of Botany, University of Dhaka. 
Anonymous, 2012b. Angiospermic Flora of Trishal Upazila, Maymenshing, M.S. Thesis (Unpublished), Plant Taxonomy and Ethnobotany Laboratory, Department of Botany, University of Dhaka.

Arefin, M.K., Rahman, M.M., Uddin, M.Z. and Hassan, M.A. 2011. Angiosperm flora of Satchari National Park, Habiganj, Bangladesh. Bangladesh J. Plant Taxon. 18(2): 117-140.

Azkar, M.A.M.B.I., Chatani, S. and Sudo, K. 2012. Simulation of urban and regional air pollution in Bangladesh. J. of Geophysical Research, Atmos. Vol. 117.

Begum, B.A., Biswas, S.K. and Hopke, P.K. 2006. Temporal variations and spatial distribution of ambient PM 2.2 and PM 10 concentrations in Dhaka, Bangladesh. Sci Total Environ. 358(1-3):36-45.

Begum, B.A., Biswas, S.K. and Hopke, P.K. 2008. Assessment of trends and present ambient concentrations of PM 2.2 and PM 10 in Dhaka, Bangladesh. Air quality. Atmos. Health. 1:125-133.

Begum, B.A., Biswas, S.K and Hopke, P.K. 2011. Key issues in controlling air pollutants in Dhaka, Bangladesh. Atmos. Environ. 45:7705-7713.

Braun-Blanquet, J. 1932. Plant Sociology (Transl. G.D. Fuller and H.S. Conrad). McGraw-Hill, New York. 539 pp.

Braun-Blanquet, J. 1964. Pflanzensoziologie, Grundzüge der Vegetationskunde. 3rd Edition, Springer-Verlag, Berlin, 631pp.

Bridson, D.M. and Forman, L. 1989. In: Bridson, D.M. and Forman, L. (eds.). The Herbarium Handbook. Royal Botanic Gardens, Kew, London, 334pp.

Cain, S.A. 1938. The Species-Area Curve. The American Midland Naturalist, 19: 573-581.

Cottam, G. and Curtis, J.T. 1956. The Use of Distance Measures in Phytosociological Sampling. Ecol. 37(3) : 451-460.

Cottam, G., Curtis, J.T. and Hale, B.W. 1953. Some Sampling Characteristics of a Population of Randomly Dispersed Individuals. Ecol. 34(4): 741-757.

Cronquist, A. 1981. An Integrated System of Classification of Flowering Plants. Columbia University Press, New York, pp. 1-1262.

Flora of North America Editorial Committee (eds.). 1993-2014. Flora of North America. Vols. 128. New York and Oxford.

Glen, S. 2016. Jaccard Index / Similarity Coefficient. From Statistics HowTo.com: Elementary Statistics for the rest of us! https://www.statisticshowto.com/jaccard-index/.

Haidari, M. 2013. Study of herb diversity in the zagros forest (Case study: Kurdistan province), Int.l J. Adv. Biol.Biomed. Res. 1(1): 25-34.

Hooker, J.D. 1872-1897. The Flora of British India. Vols. 1-7. L. Reeve \& Co., Ashford, Kent, UK.

Hossain, M.F., Elahi, S.F. and Khondaker, M. 2003. A comparison between ecological and conventional rice Farming systems in Bangladesh. Diffuse Pollution Conference Dublin, 8C Ecol. 8: 57-64.

Huq, A.M. 1986. Plants name of Bangladesh. Bangladesh National Herbarium, BARC, Dhaka, Bangladesh, pp. 1-289.

IPNI, 2019. International Plant Names Index. Published on the Internet http://www.ipni.org. The Royal Botanic Gardens, Kew, Harvard University Herbaria \& Libraries and Australian National Botanic Gardens. [Accessed on 15 March 2019].

Jaccard, P. 1912. The distribution of the flora in the alpine zone. New Phytologist. 11 (2): 37-50. Doi:10.1111/j.1469-8137.1912.tb05611.x. ISSN 0028-646X.

Jensen, R. and Peppard, D.M. 2004. The Traditional Brick making Industry and the Rural Economy of Vietnam. J. Asian and African stud. 39(3):193-207.

Khan, M.S. (eds.). 1972-1987. Flora of Bangladesh, Fasc. 1-39. Bangladesh National Herbarium, Dhaka. 
Khan, M.S. and Halim, M. 1987. Aquatic Angiosperms of Bangladesh. Bangladesh National Herbarium, BARC, Dhaka. pp. 1-120.

Khan, M.S. and Huq, A.M. 2001. The vascular flora of Chunati wildlife sanctuary in south Chittagong. Bangladesh J. Plant Taxon. 8(1): 47-64.

Khan, M.S., Rahman, M.M., Huq, A.M., Mia, M.M.K. and Hassan, M.A. 1994. Assessment of Biodiversity of Teknaf Game Reserve in Bangladesh focusing on economically and ecologically important plant species. Bangladesh J. Plant Taxon. 1(1): 21-23.

Khanam, R., Khan, S.A. and Rahim, M.A. 2020. Angiosperms in Narsingdi district of Bangladesh: Class Magnoliopsida. Bangladesh J. Plant Taxon. 27(1), 153-171. https://doi.org/10.3329/bjpt.v27i1.47576.

Krebs, C.J. 1989. Ecological methodology. New York, NY: Harper and Row Publishers Inc., 654 p.

Le, H.A. and Oanh, N.T.K. 2010. Integrated assessment of brick kiln emission impacts on air quality. Environmental Monitoring and Assessment. 171(1-4):381-394.

Nahaer, N. 2014. A taxonomic study of the Angiosperms of Birulia union of Savar upazila under Dhaka district. M.S. Thesis (Unpublished). Plant Systematics and Biodiversity Laboratory, Department of Botany, Jahangirnagar University, Savar, Dhaka-1342.

Nasir, E. and Ali, S.I. (eds.). 1980-2005. Flora of Pakistan. Vols. 1-209. University of Karachi, Karachi, Pakistan.

Pasha, M.K. and Uddin, S.B. 2013. Dictionary of Plant Names of Bangladesh (Vascular Plants). Janokalyan Prokashani, Andarkilla, Chittagong, pp 1-320.

Pichi, S.R.E.G. 1977. Tentamen pteridophytorum genera in taxonomicum ordinem redigendi. Webbia. 31:313-512.

Prain, D. 1903 (Reprint 1963). Bengal Plants. Vols. 1 and 2. Botanical Survey of India, Calcutta.

Radaei, M. and Amini, M. 2013. Study of the plant diversity in Hyrcanian forest (case study: Lajim forest, Mazandaran province, North of Iran). Int. J. Biosci.Vol. 3 (10): 273-279.

Rahman, A.H.M.M. 2013. Angiospermic flora of Rajshahi District, Bangladesh. American J. Life Sci.1(3): 105-112.

Rahman, M.O. and Hassan, M.A. 1995. Angiospermic flora of Bhawal National Park, Gazipur (Bangladesh). Bangladesh J. Plant Taxon. 2(1\&2): 47-79.

Rahman, M.O., Antara, R.T., Begum, M. and Hassan, M.A. 2012. Floristic diversity of Dhamrai upazila of Dhaka with emphasis on medicinal plants. Bangladesh J. Bot. 41(1): 71-85.

Rahman, M.O., Begum, M. and Ullah, M.W. 2013. Angiosperm flora of Sadar Upazila of Munshiganj District, Bangladesh. Bangladesh J. Plant Taxon. 20(2): 213-231.

Rashid, S.H. and Mia, M.M.K. 2001. Angiospermic flora of Madhupur National Park, Tangail, Bangladesh. Bangladesh J. Plant Taxon. 8(2): 63-82.

Raunkiaer, C. 1934. "The Life-form of plants and statistical plant geography," Claresdon Press, Oxford, UK. 632p.

Roy, G.K. and Khan, S.A. 2020. Preliminary taxonomic study on homestead flora of four districts of Bangladesh: Magnoliopsida. Bangladesh J. Plant Taxon. 27(1): 37-65. https://doi.org/10.3329/bjpt.v27i1.47567.

Sarker, K., Islam, M.R., Uddin, M.Z. and Hassan, M.A. 2013. Angiosperm flora of Manikgonj Sadar Upazila, Bangladesh. J. Asiat. Soc. Bangladesh, Sci. 39(2): 147-166. https://doi.org/10.3329/jasbs.v39i2.17851.

Shannon, C.E. 1948. A mathematical theory of communication. Bell System Technical J, 27: 379423.

Shetu, S.S., Khan, S.A. and Uddin, S.N. 2018. Checklist of Angiosperms extant in Mirpur area of Dhaka City, Jahangirnagar University J. Biol. Sci. 7(2): 47-64. 
Siddiqui, K.U., Islam, M.A., Ahmed, Z.U., Begum, Z.N.T., Hassan, M.A., Khondker, M., Rahman, M.M., Kabir, S.M.H., Ahmad, M., Ahmed, A.T.A., Rahman, A.K.A. and Haque, E.U. (eds.). 2007. Encyclopedia of Flora and Fauna of Bangladesh. Vols. 5 \& 11. Asiatic Society of Bangladesh, Dhaka, Bangladesh.

Simpson, E.M. 1949. Measurement of diversity. Nature. 163:688.

Singh, A.L. and Asgher, M.S. 2005. Impact of brick kilns on land use/landcover changes around Aligarh city, India. Habitat International. 29(3): 591-602.

Singh, H.B. and Subramaniam, B. 2008. Field Manual on Herbarium Techniques. National Institute of Science Communication and Information Resources, New Delhi, India. pp. 1297.

Singh, M. and Kumar, M. 2013. Study of plant diversity of Jind district, Haryana, India, Asian J. Plant Sci. and Res. 3(3): 44-53.

Smitinand, T. and Larsen, K. (eds.). 1975. Flora of Thailand, Vol. 2, part 3, Applied Scientific Research Corporation of Thailand, Bangkok. pp.1-280.

Smitinand, T. and Larsen, K. (eds.). 1989. Flora of Thailand, Forest Herbarium, Royal Forest Department, Bangkok. Mem. Fac. Sci. Kyoto Univ. Biol. Sci. 3(4): 67-88.

Sultana, S., Uddin, S.N., Khan, S.A. and Rahim, M.A. 2013. A taxonomic study on the flora of Jahangirnagar university campus. Bangladesh J. Plant Taxon. (in review).

The Plant List, 2013. The Plant List, a working list of all plant species. Version 1.1 <http://www.theplantlist. org/>. Accessed on 15 July 2019.

TROPICOS, 2020. Tropicos.org. <www.tropicos.org>. Missouri Botanical Garden, Saint Louis, Missouri, USA. Accessed on 10 March 2020.

Uddin, M.Z. and Hassan, M.A. 2010. Angiosperm diversity of Lawachara National Park (Bangladesh): A preliminary assessment. Bangladesh J. Plant Taxon. 17(1): 9-22.

Uddin, S.B. and Rahman, M.A. 1999. Angiospermic flora of Himchari National Park, Cox's Bazar, Bangladesh. Bangladesh J. Plant Taxon. 6 (1): 31-68.

Watson, M.F., Akiyama, S., Ikeda, H., Pendry, C.A., Rajbhandari, K.R. and Shrestha, K.K. (eds.). 2011. Flora of Nepal, Vol. 3. Magnoliaceae to Rosaceae. Royal Botanic Gardens, Edinburgh, pp.1-512.

Wu, Z.Y. and Raven, P.H. (eds.). 1994-2001. Flora of China. Vols. 8, 15-18, 24. Science Press, Beijing, and Missouri Botanical Garden Press, St. Louis.

Wu, Z.Y., Raven, P.H. and Hong, D.Y. (eds.). 1999-2013. Flora of China. Vols. 2-7, 9-14, 19-23, 25. Science Press, Beijing, and Missouri Botanical Garden Press, St. Louis. 